\title{
Selective Electron Beam Erosion and Deposition of Materials
}

\author{
David C Joy ${ }^{+}$and Philip D Rack \\ Dept. of Mat. Science and Engineering, University of Tennessee, Knoxville TN37996 \\ + and Oak Ridge National Laboratory, Oak Ridge, TN 37831
}

The fact that a beam of electrons traveling through a vacuum environment can selectively deposit or remove material from a surface has been known for more than fifty years. Early users of Electron Microprobes observed that the beam would deposit carbon on to the irradiated area [1], and Ballantyne found that by deliberately introducing selected 'precursor' gases into the beam path a variety of different metals could be deposited as desired [2]. Further research showed that other choices of gas resulted in etching of the sample surface [3]. Although Focussed Ion Beam (FIB) systems are now usually seen as the tool of choice for etching and deposition there continue to be good reasons why the alternative e-beam approach has merit. First, there is a clear financial advantage because with the addition of a gas injector any conventional SEM can be used for these purposes while still maintaining the normal imaging capabilities of the microscope. Second, electrons - unlike ions - do not result in the implantation of $\mathrm{Ga}^{+}$or other ion species into the sample, nor do they produce amorphous damage, considerations which for some applications such as sample thinning or the repair of lithographic masks are crucial.

Our experiments have been performed in variable pressure SEMs such as the Hitachi $\mathrm{S} 4300 \mathrm{SE} / \mathrm{N}$ which is equipped with a Schottky emitter and can provide beam currents up to $30 \mathrm{nA}$ at $30 \mathrm{keV}$. Gas is injected into the system through a hypodermic needle attached to a Wobble stick which allows the nozzle to be positioned in three dimensions relative to the sample. Typically the rate of gas injection, controlled by a precision needle valve, is low enough that the SEM can be operated in either high, or low, vacuum mode without any risk to the Schottky emitter. For deposition a variety of precursor gases have been examined because, in principle, any of those in CVD processing can be expected to be useful. For tungsten we now use $\mathrm{WF}_{6}$ which is a liquid at room temperature and so can readily be transferred as a vapor. With a beam energy of $10 \mathrm{keV}$ and a beam current of $\ln A$ the deposition rate is then initially of the order of $10 \mathrm{~nm}$ per minute. Because the injected gas plume is localized close to the substrate surface there is little or no beam scattering and lines as narrow as $50 \mathrm{~nm}$ can be patterned by adjusting the beam dose and scan speed. Depositions in spot or line mode (figure 1) have close to vertical sides but a characteristically rounded top profile. The deposition mechanism involves decomposition by primary and emitted secondary and backscattered electrons of precursor molecules temporarily present on the surface. The number of molecules striking the surface per unit time depends on the gas pressure, and their residence time depends on the surface binding energy and temperature. Arrhenius plots of deposition rate vs temperature show that the effective binding energy is about $130 \mathrm{meV}$. For a surface nominally at room temperature this implies that a temperature increase of only one degree $\mathrm{C}$ will cause a measurable drop in deposition rate, suggesting that the observed fall-off in deposition rate with increasing layer thickness or height can be attributed to the local increase in temperature as deposition proceeds. Lowering the temperature to $-30 \mathrm{C}$ enhances the rate by about $3 \mathrm{x}$. 
Unlike ions, electrons do not sputter material directly so erosion of a surface requires a chemically active precursor gas. A variety of choices is possible but $\mathrm{XeF}_{2}$ has been found to be the most widely useful, although water vapor by itself is able to etch carbon and carbonaceous materials. A wide variety of specimens including metals, semiconductors and inorganics have now successfully been eroded. The rate of attack depends on the sample and on the beam and gas conditions but rates of the order of tens of nanometers per minute per nanoamp are typical. The spatial resolution is comparable with that obtained in deposition, but because $\mathrm{XeF}_{2}$ will continue to etch even when the electron beam is turned off then careful control is required to achieve precision results. Successful applications have been made in areas such as the repair and editing of EUV lithography masks, nanoscale device prototyping, and as a technique for 'damage free' TEM sample preparation.

As variable pressure SEMs become more widely used it can be anticipated that electron beam erosion and deposition techniques will be recognized as being a valuable complement to the FIB, as well as providing important "value added" to the capabilities of the standard instrument.

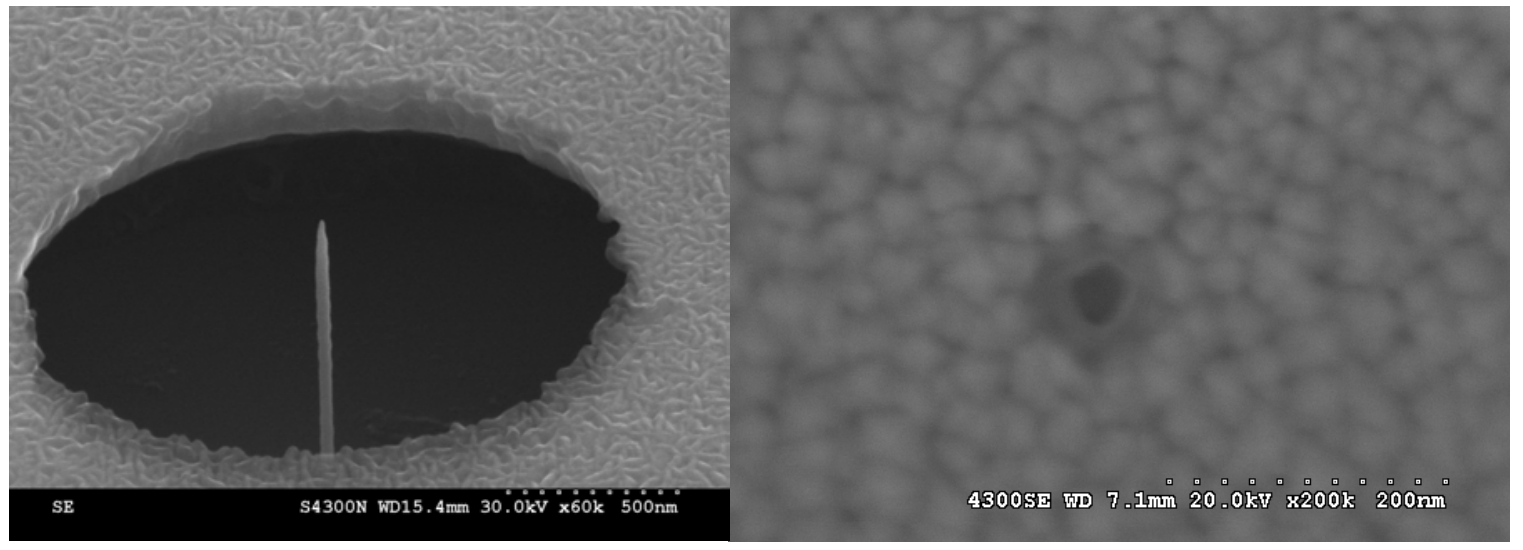

Fig.1 Electron beam deposited tungsten nanofiber for use as a cold field emission Fig.2 Electron beam induced etching of $\sim 45 \mathrm{~nm}$ tungsten grain cathode

\section{References:}

(1) R Castaing and J Deschamps, Compt. Rendu., 238, 1506, (1954)

(2) J P Ballantyne, PhD Thesis, Cambridge University 1972

(3) T Shiraiwa et al., Proc. $6^{\text {th }}$ Int Conf. Xray Optics and Microanalysis, (G Shinoda, K Kohra and T Ichinokawa), Univ. of Tokyo Press, p213 (1972)

(4) Research sponsored as a customization project for INTEL Corp. by the Semiconductor Research Corporation, monitors Dr. Ted Liang and Dr. Dan Herr. Oak Ridge National Laboratory is managed by UT-Batelle, LLC for U.S. Department of Energy under contract number DE-AC05-00OR22725 